\title{
La neurobiónica como una disciplina emergente: Aportaciones al futuro de las ciencias de la salud y las ciencias sociales
}

\author{
Parra-Bolaños, Nicolás ${ }^{1\left(^{*}\right)}$; Jaramillo Vélez, Sindy Tatiana² \& Sierra Gómez, Stefany ${ }^{2}$ \\ ${ }^{1}$ Asociación Educar para el Desarrollo Humano, Laboratorio de Neurociencias \\ y Educación, Buenos Aires, Argentina. \\ ${ }^{2}$ Tecnológico de Antioquia - Institución Universitaria, Programa de Trabajo Social, \\ Medellin, Colombia.
}

\section{RESUMEN}

La neurobiónica es una ciencia que se encuentra limitando entre las ciencias de la salud, las ingenierías y las neurociencias, tomando elementos de dichas disciplinas científicas, y llegando a configurarse como una ciencia relativamente nueva, lo que ha provocado que sea un campo muy poco conocido, aún entre los profesionales de las ciencias de la salud, sin embargo, no por ello deja de ser una disciplina que por sí misma es una ciencia básica, pero también, una ciencia aplicada. El presente estudio ha empleado dos softwares: CASP y STROBE, para hacer un sondeo por las cuarenta bases de datos de los Institutos Nacionales de la Salud de los Estados Unidos de Norteamérica, con el objeto de revisar los últimos diez años en lo referente a literatura científica sobre neurobiónica. Se analizaron seis diferentes ecuaciones para la búsqueda y se aplicaron criterios de inclusión y exclusión. El estudio arrojó información y hallazgos que indican que la neurobiónica es un campo sumamente prometedor, tanto para hacer aportaciones para las ciencias de la salud como para las ciencias sociales, dada su enorme versatilidad y capacidad de ejecución de trabajos transdisciplinarios, pues se encuentra en la frontera del conocimiento humano, hasta el punto de obligarnos a repensar la definición de lo que es la especie humana a la luz del uso de tecnologías integradas al sistema nervioso central.

Palabras clave: Neurobiónica; Ingeniería; Neurociencias; Bibliometría; Revisión

\section{Neurobionics as an Emerging Discipline: Contributions to the Future of Health Sciences and Social Sciences}

\section{ABSTRACT}

Neurobionics is a science that is bordering between the health sciences, engineering and neurosciences, taking elements from these scientific disciplines, becoming a relatively new science, which has caused it to be a very little known field, even among health science professionals, however, they are still a discipline that is itself a basic science, but also an applied science. The present study has used two softwares: CASP and STROBE, to conduct a survey of the forty databases of the National Institutes of Health of the United States of North America, in order to review the last ten years in relation to literature scientist on neurobionics. Six different equations were analyzed for the search and inclusion and exclusion criteria were applied. The study yielded information and findings that indicate that neurobionics is an extremely promising field, both for making contributions to the health sciences and for the social sciences, given its enormous versatility and capacity to carry out transdisciplinary work, since it is in the frontier of human knowledge, to the point of forcing us to rethink the definition of what the human species is in light of the use of technologies integrated into the central nervous system.

Keywords: Neurobionics; Engineering; Neurosciences; Bibliometrics; Review 


\section{INTRODUCCIÓN}

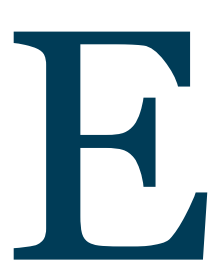

I uso de tecnologías no hace alusión solamente a modernas herramientas digitales o virtuales, sino que, la acepción más convencional identifica a la tecnología con cualquier tipo de técnica o destreza que la especie humana o cualquier otra especie pueda emplear para facilitar su vida o la vida de los miembros de su especie. Este tipo de explicación procede de principios antropológicos y arqueológicos, pues estas dos ciencias han venido estudiando el origen de los diferentes tipos de tecnologías primitivas en culturas humanas milenarias, tanto en Oriente como en Occidente. En el caso particular del presente estudio, lo que se ha hecho es una revisión bibliométrica de la forma en que han ido evolucionando las tecnologías de neuroimagen hasta llegar a permitir la confluencia de ciencias aparentemente disimiles para conseguir mejorar las técnicas de observación y análisis de epifenómenos conductuales concretos (Erdő \& Krajcsi, 2019; Payne, Furness \& Stebbing, 2019; Van-Der-Helm, Van-Der-Meer, Eijkel, Van-Den-Berg \& Segerink, 2016).

Las neurociencias junto con la neurología y las ciencias del comportamiento, han venido haciendo grandes esfuerzos en los últimos 100 años, por conseguir una mayor y mejor comprensión de la conducta humana y de otras especies, basando sus avances y desarrollos en la creación y producción masiva de nuevas tecnologías, dada la poca o nula accesibilidad que suelen presentar a nivel molecular, tanto los complejos de colonias celulares de los diferentes organismos a estudiar, como todos los demás componentes orgánicos que se correlacionan con el comportamiento, pero que, al carecer de tecnologías precisas y de gran profundidad en la medición y observación de variables, han generado que durante incontables siglos, la explicación de la conducta humana y de otras especies, tenga que estar mediada por elucubraciones y conjeturas, tanto de origen pseudocientífico como metafísico, y mágico-religioso (Jandial \& Hoshide, 2017; Sivaramakrishnan \& Madhavan, 2018).
Básicamente lo que se hará en este manuscrito, es desarrollar un análisis de los principales y más significativos progresos de la conjunción entre la ingeniería biónica y las neurociencias, lo que daría como resultado, una nueva disciplina que cada vez toma más y más fuerza en las naciones con mayor capacidad de inversión en innovación científica y tecnológica, dicha nueva disciplina se conoce como neurobiónica, por lo que se hará una revisión bibliográfica en las principales Bases de Datos médicas del mundo, más concretamente, en las cuarenta bases de datos que componen PubMed, generando con ello, una serie de conclusiones y reflexiones sobre los alcances y potencialidades de la neurobiónica para el siglo XXI (Duffley, Anderson, Vorwerk, Dorval \& Butson, 2019; Yu, 2016).

\section{MARCO TEÓRICO}

La neurobiónica ha sido definida como la ciencia que pretende, experimentalmente, la integración directa entre campos científicos como la electrónica, la biomecánica, la biónica y las neurociencias cognitivas con el sistema nervioso central, para perseguir la creación de nuevas metodologías que estén en condiciones de lograr la reparación, rehabilitación o sustitución de regiones encefálicas deterioradas en sus funciones específicas. Para conseguir esta ambiciosa meta, la neurobiónica ha tenido que repensar la posibilidad, más allá de la ciencia ficción, sobre las interfaces entre cerebro y tecnologías externas o invasivas, pues la neurobiónica persigue la construcción de sistemas de interfaz, capaces de entrelazar al cerebro con los ordenadores, lo que, por sus siglas en inglés se conoce como $\mathrm{BCl}$, que vendría siendo el vínculo que podría llegar a tener el cerebro con las computadoras a través de todo un complejo sistema de sensórica en el cuero cabelludo, intracortical o subdural por medio de electrodos del grosor de cabellos humanos, lo que es una tecnología que se evidencia fuertemente en herramientas como la estimulación cerebral profunda, o por sus siglas en inglés: DBS (Barker-Collo, Krishnamurthi, Theadom, Jones, Starkey \& Feigin, 2019; Jung, Abbas, Kuntaegowdanahalli \& Thota, 2018). 
El desarrollo de las tecnologías neurobiónicas no se ha dado aislado del resto de ciencias de la salud, sino que sus progresos han requerido de la colaboración mutua e interdisciplinaria entre distintos especialistas en campos de la medicina, las ciencias cognitivas, las ingenierías y las tecnologías de la información y la comunicación, por lo que, se han hecho necesarias, no solo grandes inversiones para financiar este tipo de estudios, sino que también se han necesitado grandes equipos multidisciplinarios, para poder traducir todos los hallazgos de $\mathrm{BCl}$ de alto rendimiento, cuando en entornos íntegramente experimentales de estudios con animales se precisa de la extrapolación de datos e información hacia los humanos, lo que hace que la neurobiónica, se constituya como una ciencia que depende de múltiples campos y áreas para sus desarrollos (Liang, Wang \& Yuan, 2018; Tröster, Jankovic, Tagliati, Peichel \& Okun, 2017).

La neurobiónica es una ciencia que ha ido evolucionando a grandes pasos a raíz de las investigaciones preliminares de Brindley y Lewin en la década de 1960. Fue en esa brillante época en la que estos dos científicos tuvieron la innovadora idea de implantar electrodos de forma superficial en la corteza cerebral de una mujer con diagnóstico de ceguera. Los resultados de dicho procedimiento invasivo, produjeron estimulación inalámbrica de los dos electrodos a base de fosfenos, generando puntos de luz que aparecían intermitentemente bajo programas de tiempo en el campo visual de la paciente, lo que hizo que esta mujer, con un cuadro de ceguera total, pudiese denotar o identificar los parches de luz tras cada impulso eléctrico, siendo ello, una verdadera revolución a nivel experimental, pues le representaría a las ciencias médicas, el nacimiento de un nuevo campo científico, capaz de aportar a condiciones que hasta ese entonces, se creían imposibles de corregir o rehabilitar (Erdő, Bors, Farkas, Bajza \& Gizurarson, 2018; Gilmore, Murgai, Nazer, Parrent \& Jog, 2019).

Este experimento pionero, fue seguido por otro estudio, no menos brillante en la década de 1970, el cual estuvo liderado por Dobelle y sus colegas, que proporcionaron una entrada eléctrica (input) a los dos electrodos implantados en la corteza visual de personas con diagnóstico de ceguera, lo que tuvo lugar a través del montaje de una cámara que se ubicó en monturas de gafas, por lo que fue un procedimiento que tuvo las dos peculiaridades clínicas de cualquier procedimiento clínico dentro del quirófano: invasivo y no invasivo, haciendo que se evidenciara la posibilidad de combinar ambas técnicas de forma exitosa para empezar a mejorar funciones que nunca antes en la historia de la humanidad se habían logrado recuperar (Bergmann, Krewer, Jahn \& Müller, 2018; Hu, Tong, Ho, Xue, Rong \& Li, 2015).

Mucho se ha oído mencionar en todos los países del mundo acerca del implante coclear, pero lo que pocos saben, es que al igual que los dos estudios antes descritos, este avance médico e ingenieril, también ha sido desarrollado por la neurobiónica, específicamente en las décadas de 1960 y 1970. Estos avances que en su momento fueron una verdadera revolución, cuando son vistos desde la óptica de las más modernas tecnologías contemporáneas de la neurobiónica, podrían parecer sumamente primitivos en el presente, pero gracias a que existieron científicos visionarios y dispuestos a romper paradigmas convencionales, es que ahora contamos con prótesis de hasta 22 canales, cuando en esos estudios pioneros a duras penas se alcanzaba a contar con un par de canales, y no solo ello, sino que los implantes cocleares, se han convertido en una de las tecnologías más ampliamente comerciales, llegando a posicionarse como éxitos rotundos en los cinco Continentes, pues son dispositivos pequeños, y sumamente portátiles como para ser transportados en las gafas de los usuarios, y tienen la capacidad de restaurar la audición en personas sordas, pero con la condicionante de que cuenten con todo su sistema de nervios auditivos, totalmente intactos o no dañados. Este tipo de avances neurobiónicos han servido para ayudar a aquellos individuos que presentan perdida de sus nervios auditivos, pues ya es posible implantarles un tipo de prótesis que posee múltiples electrodos que van desde el núcleo coclear hasta el tronco cerebral, y todo ello, de forma directa (Rabipour, Wu, Davidson \& Iacoboni, 2018; Xie, Liu, Fu, Dai, Zhou \& Lieber, 2015).

El campo de la neurobiónica ha demostrado avances para la humanidad, como los ya descritos, sin embargo, no se ha quedado solo ahí, sino que sus 
éxitos experimentales han llegado a los más altos puestos de poder en las naciones del primer mundo, posibilitando con ello, que muchos de los proyectos científicos que se vienen desarrollando desde la década de los 1960, estén reportando diversos avances, que le han proporcionado al Gobierno de los Estados Unidos y a los Institutos Nacionales de la Salud, conocido por sus siglas en inglés como: $\mathrm{NIH}$, múltiples y muy relevantes soluciones de ingeniería biónica, puestos al servicio de las miles y miles de personas en situación de discapacidad que son ciudadanos de los Estados Unidos, erigiendo a la neurobiónica, como una de las ciencias que mayores aportaciones ha realizado y continua realizando a los veteranos de guerra que han perdido uno o más de sus brazos o piernas, además de la visión y otros sentidos, permitiéndole a estas víctimas de la guerra, mejorar drásticamente su calidad de vida, gracias a los avances surgidos en el seno de la neurobiónica (Herrman, Egge, Konglund, Ramm-Pettersen, Dietrichs \& Taubøll, 2019; Lee, Kim, Kim, Lee, Chung, Kim \& Yoo, 2017).

La neurobiónica ha ido aumentando en alcance y poder explicativo debido a sus innovadores diseños para toda la gama de electrodos que se requieren en distintos procedimientos quirúrgicos de neurocirugía, además de los constantes esfuerzos por lograr cada vez más la miniaturización de circuitos electrónicos y la fabricación de los mismos con una longevidad aun mayor, lo que le ha permitido a las tecnologías inalámbricas, ir evolucionando con velocidad inusitada de la mano de un aumento exponencial de poder computacional y de procesamiento en los dispositivos creados para todo tipo de procedimientos médicos invasivos y no invasivos de alta complejidad. En esta misma línea de trabajo, los neurocientíficos especialistas en neurobiónica, han procurado mejorar la interfaz entre el cerebro humano y los electrodos, de tal modo que, los electrodos son implantados sin romper o quemar áreas encefálicas de compromiso cognoscitivo, premotor o motor, lo que representa un salto cualitativo de proporciones descomunales respecto de los procedimientos invasivos tradicionales (Chesters, Möttönen \& Watkins, 2018; Malling, Morberg, Wermuth, Gredal, Bech \&
Jensen, 2018).

La generación de una interfaz cada vez más efectiva entre cerebro y ordenadores o $\mathrm{BCl}$, es una de las proyecciones de mayor envergadura para la neurobiónica, pues ello se constituye como uno de los principales objetos de estudio para definir a esta ciencia, produciendo con ese propósito claro, un elevado número de avances que, por lo emocionante y sorprendente de sus promesas, se han vuelto virales en canales de televisión y redes sociales de todo el planeta, mostrando una simbiosis cada vez más fuerte y consolidada entre las neurociencias y las ingenierías. Para entender mucho mejor las aportaciones técnicas de la neurobiónica, es necesario tener claridad respecto de que los electrodos que se diseñan en los laboratorios de $\mathrm{BCl}$, generan huellas y registros eléctricos de la actividad sináptica de la corteza motora en los humanos, haciendo énfasis en que, para los casos de pacientes que han sufrido de alguna clase de parálisis, es posible decodificar mediante ordenadores, dichas señales eléctricas y conseguir reproducirlas, para los casos en que áreas encefálicas especializadas han dejado de tener comunicación sináptica. En esta misma línea, también existen otra gama de electrodos que son capaces de inhibir corrientes eléctricas, tal como en el caso de enfermedades neurodegenerativas como el Mal de Parkinson, entre muchas otras (Bensmaia, 2015; De-La-Peña, Fernández-Medina, Parra-Bolaños \& Martínez-Restrepo, 2016).

La robótica es otra de las disciplinas que han entrado a constituirse como campos fundamentales para la neurobiónica, pues gran parte de las más sofisticadas tecnologías de la neurobiónica han venido evolucionando de la mano de la robótica, por lo que, a la fecha, no es posible concebir a la neurobiónica como una ciencia separada de la robótica. Esta última ha venido creciendo y mejorando en el diseño e implementación de brazos robóticos para conseguir que la neurobiónica pueda restaurar los movimientos en pacientes con diferentes tipos de parálisis. La integración simultánea de $\mathrm{BCl}$ con la corteza sensorial, no solo contribuyen a mejorar aún más las destrezas sobre el control fino y grueso, sino 
que, en la última década, la neurobiónica junto a la robótica, han conseguido logros tan apabullantes como: Proporcionar capacidad de deambulación en pacientes con diagnóstico de paraplejia mediante exoesqueletos robóticos; restauración de la visión en individuos con ceguera adquirida; detección y regulación de ataques epilépticos; mejoramiento sobre los trastornos del movimiento y los trastornos de la memoria; conectividad funcional de alta fidelidad dentro de pequeños grupos de neuronas motoras por medio de implantes consistentes en microelectrodos ubicados en la corteza cerebral, junto con electrodos posicionados en la superficie cortical, las que vendrían siendo técnicas no invasivas, además de técnicas de registro en torno a los dos tipos de sinapsis (química y eléctrica), para mejorar los diagnósticos de pacientes con enfermedades neurodegenerativas (Arendsen, Hugh-Jones \& Lloyd, 2018; Elder, Colloby, Firbank, McKeith \& Taylor, 2019; Indahlastari, Albizu, Nissim, Traeger, O'Shea \& Woods, 2019).

Las tecnologías $\mathrm{BCl}$ aún se encuentran todavía en fases muy tempranas de desarrollo, por lo que la neurobiónica se puede considerar a todas luces, como una ciencia técnicamente muy nueva, que todavía aguarda por la aparición y puesta en escena de mejores técnicas ligadas a mayor número de ensayos clínicos multicéntricos, antes de que sus aplicaciones clínicas puedan aplicarse de forma mucho más amplia y con un impacto social más fuerte en cuanto a la atención y rehabilitación de funciones alteradas o dañadas en pacientes con distintos diagnósticos de discapacidad, por lo que, los desafíos que la neurobiónica debe afrontar son muy elevados y requerirán, sin lugar a dudas, del trabajo interdisciplinario y mancomunado con otras ciencias y otras ingenierías que podrían abrir nuevas fronteras de conocimiento para la neurobiónica (Eisemann, Costa, Strelau, Mittelbronn, Angel \& Peterziel, 2018; Terraneo, Leggio, Saladini, Ermani, Bonci \& Gallimberti, 2016).

Para la realización de este estudio de revisión, se han configurado seis matrices de datos, las que se han estructurado basados en las ecuaciones que aparecen en la Tabla 1, permitiendo que, por medio de los programas The Critical Appraisals Skills Programme (CASP) y el Strengthening the Reporting of Observational Studies in Epidemiology (STROBE), se analizaran todas las publicaciones ubicadas dentro de las cuarenta principales bases de datos de los Institutos Nacionales de la Salud de los Estados Unidos de Norteamérica, que también son conocidos por sus siglas en inglés como NIH. Dicho conglomerado de bases de datos se denomina como PubMed, haciendo énfasis en que se ha hecho un barrido de publicaciones de la última década, lo que quiere decir que, se han revisado documentos desde el año 2011 hasta el año 2020. Esta revisión se ha realizado el 23 de septiembre de 2020.

Tabla 1. Condensado por matrices de la bibliometría ejecutada con los softwares CASP y STROBE (2011-2020).

\begin{tabular}{c|c|c}
\hline Ecuación & Base de Datos & Total \\
\hline Transcranial & PubMed & 2,913 \\
\hline $\begin{array}{c}\text { Deep Brain } \\
\text { Stimulation }\end{array}$ & PubMed & 509 \\
\hline Brain Electrode & PubMed & 622 \\
\hline $\begin{array}{c}\text { Brain } \\
\text { Technology }\end{array}$ & PubMed & 1,540 \\
\hline Bionic & PubMed & 70 \\
\hline $\begin{array}{c}\text { Robot Assisted } \\
\text { Brain }\end{array}$ & PubMed & 55 \\
\hline
\end{tabular}

\section{Fuente: Elaboración propia (2020).}

Las ecuaciones usadas para ser procesadas con los softwares CASP y STROBE, fueron las siguientes, según se puede observar en la Tabla 1: "Transcranial", "Deep Brain Stimulation", Brain Electrode", "Brain Technology", "Bionic" y "Robot Assited Brain", debido a que son las variables que de forma directa se correlacionan con mayor fuerza en cuanto al desarrollo y la evolución de la neurobiónica como una ciencia. Las bases de datos revisadas se corresponden, como ya se mencionó con ante- 
rioridad, a las de los $\mathrm{NIH}$, arrojando como resultados generales que, para la primera ecuación se filtraron un total de 2,913 artículos; para la segunda ecuación se encontraron 509 manuscritos; para la tercera ecuación se recogieron 622 estudios; para la cuarta ecuación se condensaron 1,540 textos; para la quinta ecuación se colectaron 70 investigaciones y para la sexta ecuación se evidenciaron 55 artículos.

Los criterios de inclusión para este estudio fueron Ios siguientes: a) Que sean artículos científicos; b) Que estén indexados en los primeros cuartiles; c) Que sean ensayos clínicos y d) Que cuenten con metodologías experimentales. En tanto que, los criterios de exclusión fueron los siguientes: A) Se descartaron trabajos de grado, tesis y cartas al Editor; B) Se descartaron estudios de reflexión y estudios de revisión; C) Se descartaron estudios ex post facto, estudios cuasi-experimentales y estudios pre-experimentales y D) Se descartaron estudios con poca precisión técnica y escaso rigor metodológico. Estos criterios son un rasero sumamente efectivo para garantizar que este estudio bibliométrico cumpla con los más altos estándares de calidad en cuanto a la literatura científica empleada.

\section{RESULTADOS Y/O DISCUSIÓN}

Se tomaron 30 referencias para la construcción de este estudio, todas ellas son estrictamente, ensayos clínicos que van desde 2011 hasta 2020, por lo que, en aras de conseguir un mejor filtrado de los datos, se tomaron los datos en seis matrices distintas. Los títulos que el CASP y el STROBE permitieron recoger, para que, de ese modo, se pudiesen aplicar otros filtros de mayor rigor, fueron las palabras clave que envuelven el objeto de estudio de la neurobiónica, por lo que, este ejercicio bibliométrico se hizo de forma escalonada y sistemática hasta conseguir recoger solo los 30 estudios mencionados. Dichos manuscritos fueron seleccionados, además, por los criterios de inclusión y exclusión, y por elementos constitutivos de la neurobiónica que permitieran conjugarlos con las palabras clave encontradas en el título del presente artículo, pues se persiguió el objetivo de que la selección de textos, guardara consonancia con las aportaciones que todos y cada una de las referencias podrían hacerles a las ciencias de la salud y a las ciencias sociales desde el punto de vista de las tecnologías neurobiónicas de última generación.

Este ejercicio posibilitó reducir los 5,709 artículos registrados en la Tabla 1 a un conjunto cerrado de tan solo una treintena de estudios, los cuales fueron la columna vertebral para el diseño y la construcción de todos y cada uno de los apartados de este manuscrito, posibilitando con ello, que todo lo alusivo a la neurobiónica y su amplio abanico de posibilidades de trabajo con ciencias de la salud y con ciencias sociales se viese reflejado en la colección recogida. Por ende, no se presenta una tabla con todos y cada uno de los artículos científicos examinados, que es lo regular para este tipo de investigaciones, sino que, en aras de construir un manuscrito totalmente basado en la neurobiónica, se procedió a diseñar todas y cada una de las líneas de este estudio con respaldo teórico y metodológico devenidos de la neurobiónica, por lo que, este texto de revisión es también, una profunda reflexión de la neurobiónica en la contemporaneidad.

Autores como VanSickle, Volk, Freeman, Henry, Baldwin \& Fitzpatrick (2019), plantean en sus estudios que, el uso de electrodos y de tecnologías robóticas, podrían constituirse como uno de los pilares más sólidos para fortalecer el ejercicio y crecimiento exponencial de la neurobiónica en función de la tan anhelada transdisciplinariedad que dicha ciencia ha perseguido desde sus orígenes mismos, en tanto que, investigadores como Li, Romero-Garcia, Suckling \& Feng (2019), propenden por el uso de estudios más de carácter mixto para fortalecer a la neurobiónica en su ejercicio transdisciplinar, postura que no es compartida por los autores del presente manuscrito, dado que, la intención de la neurobiónica ha sido y es, en todo momento, articularse con las ciencias exactas y con las ciencias naturales, pues es una ciencia que nace a partir de las prácticas de más altor rigor clínico y experimental, y en menor medida, con las ciencias cognitivas y las ciencias del 
comportamiento, postura que compartimos y que guarda consonancia con la naturaleza y prioridades de la neurobiónica. Ello no quiere decir que la neurobiónica descarte de plano a las ciencias que son más de corte cualitativo, sino que su enfoque, ha sido marcadamente cuantitativo y su ejercicio profesional se ha venido desarrollando dentro del laboratorio, por lo que, su fuerza radica mayormente en el ámbito clínico y experimental.

\section{CONCLUSIONES}

La llegada de la neurobiónica, presupone que si se quiere mejorar la calidad de vida de personas con distintos tipos de discapacidad física, sensorial o con enfermedades de carácter neurológico, deben crearse laboratorios dotados con las más sofisticadas tecnologías, pues la neurobiónica, es una ciencia que está en condiciones de crear tecnologías dispuestas a asumir los más complejos desafíos que impongan los diferentes cuadros clínicos que requieran de esta ciencia para la rehabilitación de funciones que no solo dependan de la fisioterapia, fonoaudiología, neuropsicología, entre otras disciplinas que no han logrado tener muchos desarrollos tecnológicos más allá de la generación de instrumentos de evaluación y diagnóstico.

Apostar por la inclusión es una de las variables políticas y sociales de mayor importancia para el desarrollo de una nación, tanto si estamos hablando de países desarrollados como de aquellos que apenas están en vías de desarrollo. Es en esta apuesta por la inclusión, en donde el ejercicio profesional de los científicos dedicados a la neurobiónica, cobra mayor relevancia, pues son miles y miles de personas alrededor del mundo que están requiriendo prótesis y órtesis que sean livianas, prácticas, simples en su operatividad, pero capaces de generar interfaz entre el cuerpo y los dispositivos electrónicos basados en redes neuronales, de modo que cada paciente, perciba que, el instrumento biomecánico que está reemplazando su real parte del cuerpo faltante, pueda ser interpretada por el cerebro, como uno más de sus miembros u órganos, logrando con ello, una verdadera inclusión y neurorrehabilitación en todos los sujetos tratados.
Finalmente, la neurobiónica presupone que las poblaciones con daño cerebral podrían llegar a tener acceso a implantes invasivos o no invasivos, dependiendo de la complejidad de cada caso, pero siempre, teniendo en cuenta la relevancia máxima que tiene para la neurobiónica las decisiones tomadas por los Comités de Bioética, ya que para esta ciencia, desde sus mismos inicios, se ha comprendido que integrar tecnologías al cuerpo humano de forma permanente, amerita no solo diálogos, discusiones, debates y prospectivas éticas, sino también, poder generar espacios filosóficos y epistemológicos de sus avances, ya que, como toda ciencia, no puede ser ajena o estar desconectada de los principios y fundamentos que las dos disciplinas anteriormente mencionadas condicionan para todos los campos, pues la neurobiónica es de las ciencias que más controversias causará en el futuro cercano, dado que la integración entre tecnología e implantes altamente sofisticados que involucren a las funciones corticales, hace que debamos reconsiderar $y$ revaluar la definición misma del ser humano, pues nuestra evolución no está tomando un curso íntegramente natural, sino que ahora se vislumbra cada vez más, un panorama marcado por los avances tecnológicos integrados a nuestros cuerpos como parte inseparable de nuestros ciclos vitales. 


\section{REFERENCIAS}

Arendsen, L. J., Hugh-Jones, S., \& Lloyd, D. M. (2018). Transcranial Alternating Current Stimulation at Alpha Frequency Reduces Pain When the Intensity of Pain is Uncertain. The Journal of Pain: Official Journal of the American Pain Society, 19(7), 807-818. https://doi.org/10.1016/j.jpain.2018.02.014

Barker-Collo, S., Krishnamurthi, R., Theadom, A., Jones, K., Starkey, N., \& Feigin, V. (2019). Incidence of stroke and traumatic brain injury in New Zealand: contrasting the BIONIC and ARCOS-IV studies. The New Zealand Medical Journal, 132(1502), 40-54.

Bensmaia, S. J. (2015). Biological and bionic hands: natural neural coding and artificial perception. Philosophical transactions of the Royal Society of London. Series B, Biological Sciences, 370(1677), 20140209.

https://doi.org/10.1098/rstb.2014.0209

Bergmann, J., Krewer, C., Jahn, K., \& Müller, F. (2018). Robot-assisted gait training to reduce pusher behavior: A randomized controlled trial. Neurology, 91(14), e1319-e1327.

https://doi.org/10.1212/WNL.0000000000006276

Chesters, J., Möttönen, R., \& Watkins, K. E. (2018). Transcranial direct current stimulation over left inferior frontal cortex improves speech fluency in adults who stutter. Brain: A Journal of Neurology, 141(4), 1161-1171.

https://doi.org/10.1093/brain/awy011

De-La-Peña, C., Fernández-Medina, J. M., Parra-Bolaños, N., \& Martínez-Restrepo, Ó. A. (2016). Neuropsychological study in patients with Parkinson's disease: the effects of deep brain stimulation. Revista de Neurologia, 62(4), 152-156.

De-La-Peña, C., Fernández-Medina, J. M., Parra-Bolaños, N., \& Martínez-Restrepo, Ó. A. (2016). Neuropsychological study in patients with Parkinson's disease: the effects of deep brain stimulation. Revista de Neurologia, 62(4), 152-156.

Chesters, J., Möttönen, R., \& Watkins, K. E. (2018). Transcranial direct current stimulation over left inferior frontal cortex improves speech fluency in adults who stutter. Brain: A Journal of Neurology, 141(4), 1161-1171.
Duffley, G., Anderson, D. N., Vorwerk, J., Dorval, A. D., \& Butson, C. R. (2019). Evaluation of methodologies for computing the deep brain stimulation volume of tissue activated. Journal of Neural Engineering, 16(6), 066024.

https://doi.org/10.1088/1741-2552/ab3c95

Eisemann, T., Costa, B., Strelau, J., Mittelbronn, M., Angel, P., \& Peterziel, H. (2018). An advanced glioma cell invasion assay based on organotypic brain slice cultures. BMC Cancer, 18(1), 103. https://doi.org/10.1186/s12885-018-4007-4

Elder, G. J., Colloby, S. J., Firbank, M. J., McKeith, I. G., \& Taylor, J. P. (2019). Consecutive sessions of transcranial direct current stimulation do not remediate visual hallucinations in Lewy body dementia: a randomised controlled trial. Alzheimer's Research \& Therapy, 11(1), 9.

https://doi.org/10.1186/s13195-018-0465-9

Erdő, F., \& Krajcsi, P. (2019). Age-Related Functional and Expressional Changes in Efflux Pathways at the Blood-Brain Barrier. Frontiers in Aging Neuroscience, 11, 196. https://doi.org/10.3389/fnagi.2019.00196

Erdő, F., Bors, L. A., Farkas, D., Bajza, Á., \& Gizurarson, S. (2018). Evaluation of intranasal delivery route of drug administration for brain targeting. Brain Research Bulletin, 143, 155-170.

https://doi.org/10.1016/j.brainresbull.2018.10.009

Gilmore, G., Murgai, A., Nazer, A., Parrent, A., \& Jog, M. (2019). Zona incerta deep-brain stimulation in orthostatic tremor: efficacy and mechanism of improvement. Journal of Neurology, 266(11), 2829-2837.

çhttps://doi.org/10.1007/s00415-019-09505-8

Herrman, H., Egge, A., Konglund, A. E., Ramm-Pettersen, J., Dietrichs, E., \& Taubøll, E. (2019). Anterior thalamic deep brain stimulation in refractory epilepsy: A randomized, double-blinded study. Acta Neurologica Scandinavica, 139(3), 294-304.

https://doi.org/10.1111/ane.13047

Hu, X. L., Tong, R. K., Ho, N. S., Xue, J. J., Rong, W., \& Li, L. S. (2015). Wrist Rehabilitation Assisted by an Electromyography-Driven Neuromuscular Electrical Stimulation Robot After Stroke. NeuroRehabilitation and neural repair, 29(8), 767-776. https://doi.org/10.1177/1545968314565510 
Indahlastari, A., Albizu, A., Nissim, N. R., Traeger, K. R., O'Shea, A., \& Woods, A. J. (2019). Methods to monitor accurate and consistent electrode placements in conventional transcranial electrical stimulation. Brain Stimulation, 12(2), 267-274.

https://doi.org/10.1016/j.brs.2018.10.016

Barker-Collo, S., Krishnamurthi, R., Theadom, A., Jones, K., Starkey, N., \& Feigin, V. (2019). Incidence of stroke and traumatic brain injury in New Zealand: contrasting the BIONIC and ARCOS-IV studies. The New Zealand Medical Journal, 132(1502), 40-54.

Jung, R., Abbas, J. J., Kuntaegowdanahalli, S., \& Thota, A. K. (2018). Bionic intrafascicular interfaces for recording and stimulating peripheral nerve fibers. Bioelectronics in Medicine, 1(1), 55-69.

https://doi.org/10.2217/bem-2017-0009

Lee, W., Kim, S., Kim, B., Lee, C., Chung, Y. A., Kim, L., \& Yoo, S. S. (2017). Non-invasive transmission of sensorimotor information in humans using an EEG/focused ultrasound brain-to-brain interface. Plos One, 12(6), e0178476.

https://doi.org/10.1371/journal.pone.0178476

Li, J., Romero-Garcia, R., Suckling, J., \& Feng, L. (2019). Habitual tea drinking modulates brain efficiency: evidence from brain connectivity evaluation. Aging, 11(11), 3876-3890.

https://doi.org/10.18632/aging.102023

De-La-Peña, C., Fernández-Medina, J. M., Parra-Bolaños, N., \& Martínez-Restrepo, Ó. A. (2016). Neuropsychological study in patients with Parkinson's disease: the effects of deep brain stimulation. Revista de Neurologia, 62(4), 152-156.

Liang, Y., Wang, L., \& Yuan, T. F. (2018). Targeting Withdrawal Symptoms in Men Addicted to Methamphetamine With Transcranial Magnetic Stimulation: A Randomized Clinical Trial. JAMA Psychiatry, 75(11), 1199-1201.

Malling, A., Morberg, B. M., Wermuth, L., Gredal, O., Bech, P., \& Jensen, B. R. (2018). Effect of transcranial pulsed electromagnetic fields (T-PEMF) on functional rate of force development and movement speed in persons with Parkinson's disease: A randomized clinical trial. PloS One, 13(9), e0204478. https://doi.org/10.1371/journal.pone. 0204478
Payne, S. C., Furness, J. B., \& Stebbing, M. J. (2019). Bioelectric neuromodulation for gastrointestinal disorders: effectiveness and mechanisms. Nature Reviews. Gastroenterology \& Hepatology, 16(2), 89-105.

https://doi.org/10.1038/s41575-018-0078-6

Rabipour, S., Wu, A. D., Davidson, P., \& lacoboni, M. (2018). Expectations may influence the effects of transcranial direct current stimulation. Neuropsychologia, 119, 524-534.

https://doi.org/10.1016/j.neuropsychologia.2018.09.005

Sivaramakrishnan, A., \& Madhavan, S. (2018). Absence of a Transcranial Magnetic Stimulation-Induced Lower Limb Corticomotor Response Does Not Affect Walking Speed in Chronic Stroke Survivors. Stroke, 49(8), 2004-2007. https://doi.org/10.1161/STROKEAHA.118.021718

Terraneo, A., Leggio, L., Saladini, M., Ermani, M., Bonci, A., \& Gallimberti, L. (2016). Transcranial magnetic stimulation of dorsolateral prefrontal cortex reduces cocaine use: A pilot study. European Neuropsychopharmacology: The Journal of The European College of Neuropsychopharmacology, 26(1), 37-44.

https://doi.org/10.1016/j.euroneuro.2015.11.011

Terraneo, A., Leggio, L., Saladini, M., Ermani, M., Bonci, A., \& Gallimberti, L. (2016). Transcranial magnetic stimulation of dorsolateral prefrontal cortex reduces cocaine use: A pilot study. European Neuropsychopharmacology: The Journal of The European College of Neuropsychopharmacology, 26(1), 37-44. https://doi.org/10.1016/j.euroneuro.2015.11.011

Tröster, A. I., Jankovic, J., Tagliati, M., Peichel, D., \& Okun, M. S. (2017). Neuropsychological outcomes from constant current deep brain stimulation for Parkinson's disease. Movement Disorders: Official Journal of The Movement Disorder Society, 32(3), 433-440. https://doi.or$\mathrm{g} / 10.1002 / \mathrm{mds} .26827$

Van-Der-Helm, M. W., Van-Der-Meer, A. D., Eijkel, J. C., Van-Den-Berg, A., \& Segerink, L. I. (2016). Microfluidic organ-on-chip technology for blood-brain barrier research. Tissue Barriers, 4(1), e1142493.

https://doi.org/10.1080/21688370.2016.1142493 
VanSickle, D., Volk, V., Freeman, P., Henry, J., Baldwin, M., \& Fitzpatrick, C. K. (2019). Electrode Placement Accuracy in Robot-Assisted Asleep Deep Brain Stimulation. Annals of Biomedical Engineering, 47(5), 1212-1222. https://doi.or$\mathrm{g} / 10.1007 / \mathrm{s} 10439-019-02230-3$

Xie, C., Liu, J., Fu, T. M., Dai, X., Zhou, W., \& Lieber, C. M. (2015). Three-dimensional macroporous nanoelectronic networks as minimally invasive brain probes. Nature Materials, 14(12), 1286-1292.

https://doi.org/10.1038/nmat4427

Yu, S. (2016). New challenge for bionics--brain-inspired computing. Zoological Research, 37(5), 261-262.

https://doi.org/10.13918/j.issn.2095-8137.2016.5.261 